\title{
Öffentlichkeit und Narrativität bei Hannah Arendt ${ }^{1}$
}

\author{
Csaba Olay
}

Vorliegender Beitrag versucht, den systematischen Zusammenhang zwischen dem Konzept der Öffentlichkeit und dem der narrativen Struktur des Handelns im Werk von Hannah Arendt zu rekonstruieren und kritisch zu prüfen. In einem ersten Schritt kommt es darauf an, den deskriptiven Gehalt dieses Konzeptes bei Arendt zu skizzieren. Besondere Aufmerksamkeit wird dabei der eigentümlichen Struktur des öffentlichen Raumes gewidmet, die Arendt zufolge mit der Konstitution eines politischen Gebildes zusammenhängt. In einem zweiten Schritt wird die Verknüpfung von Öffentlichkeit und narrativer Struktur erörtert, vor allem unter dem Gesichtspunkt, wie die prominente Stellung der Narrativität bei Arendt dargestellt und begründet wird. Dabei wird die These vertreten, dass der öffentliche Raum und die narrative Struktur für Hannah Arendt aus verschiedenen und teilweise heterogenen Gründen grundlegend sind, ohne dass sie diese Aspekte hinreichend getrennt hätte. Dementsprechend sollen schrittweise die Problemkomplexe der Öffentlichkeit als Wesensbestimmung des Politischen und danach der Narrativität behandelt und dabei folgende Thesen plausibel gemacht werden: 1. Das Übergewicht der Öffentlichkeit in der Bestimmung des Politischen drängt instrumentelle Aufgaben in den Hintergrund. 2. Arendt kommt es aus strukturellen Gründen nicht darauf an, die narrative Zugänglichkeit eines jeden zur eigenen Identität zu klären, sondern vielmehr darauf, wie beispielhafte Taten und Leben innerhalb eines öffentlichen Raumes narrativ aufbewahrt und erinnert werden können.

1 | Vorliegender Aufsatz wurde in der MTA-ELTE Forschungsgruppe Hermeneutik ausgearbeitet und von einem Bolyai-Forschungsstipendium der Ungarischen Akademie der Wissenschaften sowie vom Projekt 81997 des ungarischen Wissenschaftlichen Landesforschungsfonds (OTKA) gefördert. 


\section{1. Öffentlichkeit als Wesensbestimmung des Politischen}

Für Hannah Arendt bildet die Öffentlichkeit im Sinne eines öffentlichen Raums die Fundamentalbestimmung des Politischen. Allein vom öffentlichen Raum her lässt sich das, was wir Politik nennen, verstehen. Der öffentliche Raum seinerseits hat Arendt zufolge notwendigerweise mit der Pluralität derer zu tun, die in ihm erscheinen, vergangene und künftige Personen mit inbegriffen, und derart besagt der Gedanke auch, dass die Politik als Erscheinung der Pluralität zu fassen ist. Im Hinblick auf die Pluralität habe Arendt bereits seit 1950 die These vertreten, könnte man ferner anführen, dass die Politik auf der Tatsache der Pluralität der Menschen beruht. ${ }^{2}$ Die Politik ist so gesehen eine, wenn auch wohl nicht die einzige Erscheinung der Pluralität menschlicher Wesen. Die Pluralität jedoch lässt sich, wie Arendt selber tut, derart mit dem öffentlichen Raum verbinden, dass die fundamentale Rolle beider für die Politik einsichtig wird. Es bleibt von vornherein zu betonen, dass in diesem Ansatz die fundamentale Vorentscheidung liegt, der zufolge die so verstandene Politik nicht, zumindest nicht primär auf die Bewältigung von vorgegebenen Aufgaben gleichwelcher Art bezogen werden soll. ${ }^{3}$ Das Politische ist also für Arendt eher eine Dimension, die unser Leben bereichert und die auch, unter bestimmten Umständen, bedroht werden bzw. verschwinden kann. Indem Hannah Arendt diese Zusammenhänge nicht explizit thematisiert, erschwert sie die positive Anknüpfung an ihr Konzept, wie darauf noch zurückzukommen sein wird, in zumindest zwei Hinsichten: zum einen bleibt dadurch der Status politischer Tätigkeit innerhalb der Hierarchie menschlicher Tätigkeiten unklar, zum andern wird dadurch der Bezug des Politischen, das von Arendt als ein Selbstzweck bestimmt wird, zum offenbar instrumentellen politischen Handeln mangelhaft beleuchtet. ${ }^{4}$

Um das zu verdeutlichen, sollen zumindest zwei Aspekte geklärt werden: zum einen soll erläutert werden, warum das Öffentliche eine Wesensbestimmung des Politischen sein sollte. Zum anderen soll die Möglichkeit der Abgrenzung eines solchen Raums, d.h. die Möglichkeit seiner Unterscheidung von anderen Räumen oder Feldern in Grundzügen verdeutlicht werden. Hannah Arendt geht von der normativen Voraussetzung aus, dass es in der Politik um Freiheit, und zwar um politische Freiheit geht: »Der Sinn von Politik ist Freiheit«, heißt es bei ihr lapidar. ${ }^{5}$ Es handelt sich dabei, genauer genommen, um eine in der Öffentlichkeit sich manifestierende Freiheit, und das ist mit der Präzisierung in Richtung der politischen Freiheit gemeint. Dieser Ausgangspunkt versteht sich jedoch kei-

2 | Arendt: Was ist Politik, S. 9. Vgl. auch: "Politik handelt von dem Zusammen- und Miteinander-Sein der Verschiedenen."(Ebd.)

3 | Deswegen spricht Arendt manchmal an einigen Stellen vom Politischen als etwas, was aus der Welt verschwinden könnte (ebd., S. 13).

4 | Siehe dazu zusammenfassend Villa: Public Freedom, S. $338 \mathrm{ff}$.

5 | Arendt: Was ist Politik, S. 28. 
neswegs. Einerseits wird damit das Politische implizit als Erscheinungsort eines grundsätzlichen Wertes bzw. einer Grundbestimmung des Menschen genommen. Andererseits lässt sich fragen, wie das Gemeinwohl, das auf naheliegende Weise als ein wesentlicher Faktor der Politik verstanden werden könnte, in diese Konzeption der Öffentlichkeit integriert oder eingegliedert werden kann. Was den ersten Punkt angeht, findet man bei Arendt nichts weiter als eher sporadische als systematische Berufungen auf eine nicht näher charakterisierte republikanische Tradition. Die nicht eigens thematisierte Anlehnung an die republikanische Tradition kommt zum Beispiel in der folgenden Passage aus einem unveröffentlichten Manuskript klar zum Vorschein, die von Machiavelli, Montesquieu und Tocqueville handelt: »They never ask: what the end of the politics, what is the end of government, for this is what they take for granted, that the political life is the best life. It cannot have an end, a goal that would be higher than itself. ${ }^{6}$ Arendt geht meines Wissens nirgends näher darauf ein. Ähnliche Passagen ließen sich mit Blick auf die Hochachtung der politischen Tätigkeit in der griechischen Polis anführen, die jedoch die allgemeine Gültigkeit dieser Bewertungen nur nahelegen, aber nicht eigens begründen. Die Art und Weise, wie Arendt den Zusammenhang von Freiheit und Öffentlichkeit in ihrem Konzept bestimmt, verdeutlicht auch die wichtigsten Punkte in Arendts Anknüpfung an eine republikanische Tradition.

Was den öffentlichen Raum angeht, unterscheidet Arendt zwei Bedeutungen des Ausdrucks »öffentlich«, die miteinander zusammenhängen, wobei für sie die zweite Bedeutung maßgebend ist. Zum einen bedeutet das Adjektiv, dass etwas für jedermann zugänglich ist, dass es gesehen und gehört werden kann. Diese Art von Öffentlichkeit bildet für Menschen ein schwerwiegendes Indiz der Realität dessen, was derart öffentlich ist. Zum anderen ist das »Öffentliche« die Welt selbst, insofern uns gemeinsam ist und »als solches sich von dem unterscheidet, was uns privat zu eigen ist, also dem Ort, den wir unser Privateigentum nennen. ${ }^{7}$ Die so verstandene Welt ist sowohl »ein Gebilde von Menschenhand wie der Inbegriff aller nur zwischen Menschen spielenden Angelegenheiten, die handgreiflich in der hergestellten Welt zum Vorschein kommen. ${ }^{8}$ Für die Akzentuierung, die Arendt hier vornimmt, ist es wesentlich, dass der maßgebende Sinn von Öffentlichkeit nicht auf der Zugänglichkeit für jedermann beruht, sondern auf der Abgrenzung gegen unsere privaten Angelegenheiten, die in ihren Ausführungen über den Gegensatz von Haushalt und öffentlichem Raum erläutert wird. Deswegen ist es ein Missverständnis, wenn man im Anschluss an Ottfried Höffe bezweifelt ${ }^{9}$, dass der Markt zur Sphäre des Haushalts gehört, weil der Warentausch sich öffentlich vollziehe und so die Welt der Wirtschaft öffentlichen Charakter besitze. Zum einen versteht Höffe hier das Adjektiv »öffentlich« im

6 | Zit. nach Breier: Hannah Arendt, S. 163.

7 | Arendt: Vita activa, S. 65.

8 | Ebd.

9 | Höffe: Politische Ethik im Gespräch, S. 24. 
Sinne der Zugänglichkeit für jedermann, also anders als Arendt. Zum anderen macht das Vorhandensein vorgegebener Ziele, und zwar privater Art, den Markt unfrei, wogegen der öffentliche Raum, wie Arendt ihn versteht, eo ipso frei ist.

Der Haushalt ist Arendt zufolge der Ort der Erhaltung des Lebensprozesses des Einzelnen. Damit wird die Privatsphäre nicht als Bereich jener Angelegenheiten verstanden, die andere nicht angehen, sondern funktional von der Notwendigkeit her, den Lebensprozess zu erhalten. Im Gegensatz zur bloßen Lebenserhaltung hat der öffentliche Raum für Arendt in erster Linie die Funktion, die Taten einzelner Menschen gegen die Vergänglichkeit zu sichern. Diese Bestimmung wird vom Modell der Polis abgelesen, die »für die Griechen - wie die res publica für die Römer - primär eine Garantie gegen die Vergeblichkeit und Vergänglichkeit des Lebens der Einzelnen [war], der Raum nämlich, der gegen alles nur Vergängliche geschützt und dem relativ Dauerhaften vorbehalten, also geradezu dafür bestimmt war, sterblichen Menschen Unsterblichkeit zu gewähren.« (VA, 70) Diese Leistung der Öffentlichkeit macht sowohl möglich, dass man sich als vortrefflich auszeichnen kann, als auch die Erfassung der Einzigartigkeit des Einzelnen mithilfe einer Lebensgeschichte ${ }^{10}$ - worauf noch zurückzukommen sein wird. Damit bekommt aber der öffentliche Raum einen antiinstrumentellen Akzent: Vortrefflichkeit und Auszeichnung gegen andere und vor anderen lassen sich nicht als Mittel zum Zweck, sondern nur als Selbstzweck begreifen.

Wie man auch die Bedeutung des öffentlichen Raums für die Erfassung der personalen Individualität bestimmen mag, ist es entscheidend, dass die Politik nach Arendts Grundüberzeugung als das Schicksal des öffentlichen Raums bestimmt werden kann. Darin spielt die Eigentümlichkeit von Arendts Bestimmung der Öffentlichkeit insofern eine Rolle, als ihr zufolge nicht nur zeitgenössische Personen, sondern auch die aus vergangenen wie auch künftigen Epochen an derselben Öffentlichkeit teilhaben: »Nur die Existenz eines öffentlichen Raumes in der Welt und die in ihm erfolgende Verwandlung von Objekten in eine Dingwelt, die Menschen versammelt und miteinander verbindet, ist auf Dauerhaftigkeit angewiesen. Eine Welt, die Platz für die Öffentlichkeit haben soll, kann nicht nur für eine Generation errichtet oder nur für die Lebenden geplant sein; sie muß die Lebensspanne sterblicher Menschen übersteigen. «" Arendt hält »dies Übersteigen in eine mögliche irdische Unsterblichkeit« für eine unerlässliche Voraussetzung sowohl der Politik wie auch einer gemeinsamen Welt und einer Öffentlichkeit. Demnach ist die Öffentlichkeit im Sinne Arendts ein gemeinsamer Raum für verschiedene Zeiten, wobei eine Schwierigkeit dieser Auffassung leicht in die

10 | Endre Szécsényi weist in seinem Aufsatz "Remarks on Hannah Arendt's Political Phenomenology" darauf hin, dass Arendt "das gesellige Jahrhundert" mit seinen verschiedenen Formen der guten Gesellschaft in ihrer Verfallsgeschichte des Politischen vernachlässigt hat, was als eine neue Form einer politischen Öffentlichkeit hätte begriffen werden können.

11 | Arendt: Vita activa, S. 68. 
Augen springt: Ihr liegt die Voraussetzung der zeitlichen Unwandelbarkeit der Öffentlichkeit zugrunde, die zwar ein Kommen und Gehen von Generationen der Handelnden erlaubt, aber die Grundstruktur des öffentlichen Raums für konstant hält.

Wie dem auch sei, Arendt sieht deutlich, es gebe keine Garantie dafür, dass es einen solchen Raum jederzeit und überall gibt: »Ein Erscheinungsraum entsteht, wo immer Menschen handelnd und sprechend miteinander umgehen; als solcher liegt er vor allen ausdrücklichen Staatsgründungen und Staatsformen, in die er jeweils organisiert wird. [...] Er liegt in jeder Ansammlung von Menschen potentiell vor, aber eben nur potentiell; er ist in ihr weder notwendigerweise aktualisiert, noch für immer oder auch nur für eine bestimmte Zeitspanne gesichert. «12 Letztendlich lässt sich aber der öffentliche Raum nicht aus sich selbst oder aus dem Gegensatz zum Verhüllten und Geheim-Gehaltenen, sondern nur aus dem Gegensatz zum Bereich des Privaten entfalten. Mehr noch: wenn gilt, dass es diesen Raum nicht jederzeit gibt, dann müsste wohl das Private insofern einen Vorrang haben, als es immer da sein muss, da es etwas mit der Erhaltung des Lebens zu tun hat. Man sollte das Verhältnis beider vermutlich eher so deuten, dass das Private und das Öffentliche zusammengehören und erst gegeneinander profiliert werden können. Arendt trennt diese Bereiche aufgrund der Funktionen, die ihnen ihr zufolge zukommen. Das Private als der Bereich der Erhaltung des Lebensprozesses diktiert eine eigentümliche Notwendigkeit, beim Eintritt in den öffentlichen Bereich wird man dagegen von der Last der Notwendigkeiten der Lebenserhaltung entbunden, und man kann in diesem Sinne frei handeln. Derart wird der öffentliche Raum als Bereich der ausdrücklichen Freiheit im Gegensatz zum Haushalt als Ort der Realisierung der für die Lebenserhaltung erforderlichen Ziele gefasst. ${ }^{13}$ An dieser Stelle wird besonders greifbar, dass Arendt die Öffentlichkeit nicht oder zumindest nicht nur aufgrund einer wie auch immer bestimmten Gesamtheit öffentlicher Angelegenheiten deutet, sondern als Freiheit im Sinne von Freiheit von den Lebensnotwendigkeiten. Die eigentümliche Ungebundenheit derer, die in den öffentlichen Raum eintreten, ermöglicht auch ihre Gleichheit, also den Umstand, dass sie von niemandem beherrscht werden und sie einander für gleich halten. Diese Ungebundenheit folgt, genauer gesehen, aus der Abwesenheit von Zielen, weil die Verfolgung von Zielen diejenigen hierarchisiert, die daran teilnehmen. So wird das Konzept des öffentlichen Raums für Arendt eine Rahmentheorie des politischen Handelns, ohne spezifische inhaltliche Prinzipien oder Aufgaben vorzuschreiben. Der Gedanke des öffentlichen Raums bestimmt nur den eigentümlich strukturierten Ort, wo inhaltliche Prin-

12 | Ebd., S. 251.

13 | Für eine kritische Diskussion der Arendtschen Auffassung des Privaten siehe Rössler: Der Wert des Privaten. Gábor Kovács bezieht Arendts Kritik des Prozess-Denkens auf die Trennung vom Öffentlichen und Privaten. Kovács: H. Arendt's Interpretation, S. 97. 
zipien vertreten werden oder eine Rolle spielen können, ohne dass die Existenz eines solchen Raums garantiert sein könnte. ${ }^{14}$

Mehrfach wurde Arendts Auffassung mit der Kritik konfrontiert, dass sie jede Art von Fachkenntnis (Versorgungsfragen, ökonomische, finanzielle Probleme) aus der politischen Tätigkeit auszuschließen scheint oder zumindest nicht imstande ist, solche Probleme zu berücksichtigen. Dabei sollte aber beachtet werden, dass Arendt diese Fragen keineswegs aus dem Auge verliert, sie betrachtet aber sie als solche, die aufgrund von erst in der Öffentlichkeit auszudiskutierenden Prinzipien zu behandeln sind. Die Philosophin vergisst nicht die Auffassung der Politik als Verwaltung der Lebensnotwendigkeiten, vielmehr ordnet sie dieses Verständnis, an das wir uns in den letzten Jahrzehnten sehr gewöhnt haben, einem anderen unter, das das Politische auf nicht instrumentelle Weise zu fassen sucht. Damit hängt auch klar zusammen, dass Arendt ein rein instrumentelles Verständnis des Politischen abweist: »Die Griechen wußten aus eigenster Erfahrung, daß ein vernünftiger Tyrann (das, was wir einen aufgeklärten Despoten nennen) für das schiere Wohlergehen der Stadt und die Blüte von materiellen und intellektuellen Künsten von großem Vorteil war. Nur mit der Freiheit war es dann vorbei. $\ll^{15}$

In einem tatsächlich stattgefundenen Gespräch reagierte sie auf den erwähnten Einwand mit der Unterscheidung von Diskussionsfragen und Verwaltungsfragen. Arendt wies darauf hin, dass es Fälle gibt, in denen man die richtigen Maßnahmen errechnen kann. »Diese Dinge können wirklich verwaltungsmäßig erledigt werden und sind dann nicht mehr Gegenstand öffentlicher Debatten. Die öffentliche Debatte kann nur Dinge behandeln, die wir - wenn wir es negativ formulieren wollen - nicht mit Sicherheit errechnen können. ${ }^{16}$ Die Strategie von Arendt bestand hier darin, vom Politischen nur eine negative Bestimmung zu formulieren, d.h. das Politische vom Fachwissen abzugrenzen. Politisch im Sinne des Gegenstandes öffentlicher Debatte ist, worüber kein Fachwissen möglich ist. Offensichtlich ist jedoch die Schwäche dieser Bestimmung, da nicht alles,

14 | Dazu gehört auch die Richtigstellung, die Arendt in der Deutung der Bestimmung des Menschen als zóon politikon bei Aristoteles vornimmt: "Aristoteles, für den das Wort ıpolitikon ، durchaus ein Adjektiv der Polis-Organisation und nicht eine beliebige Bezeichnung für menschliches Zusammenleben überhaupt war, meinte keineswegs, daß alle Menschen politisch seien oder daß es Politik, nämlich eine Polis, überall gäbe, wo Menschen lebten. Aus seiner Definition waren nicht nur Sklaven ausgeschlossen, sondern auch die Barbaren asiatischer, despotisch regierter Reiche, an deren Menschsein er keineswegs zweifelte. Was er meinte, war lediglich, daß es eine Eigentümlichkeit des Menschen ist, daß er in einer Polis leben kann und daß diese Polis-Organisation die höchste Form menschlichen Zusammenlebens darstellt und daher in einem spezifischen Sinne menschlich ist" Arendt: Was ist Politik, S. 37.

15 | Ebd., S. 41

16 | Arendt: Ich will verstehen, S. 89. 
worüber Fachwissen unmöglich oder zumindest sehr begrenzt möglich zu sein scheint - etwa ästhetische Fragen -, dem Politischen gehört. Man kann Arendt auch so verstehen, dass der Akzent auf den öffentlichen Charakter der Debatte gelegt wird, aber auch dann müsste sich angeben lassen, womit die Gegenstände der öffentlichen Diskussion zu tun haben, um sich als solche qualifizieren zu können. Es bedarf also einer näheren Charakterisierung dessen, was das Politische konstituiert, und diese Frage erhält im Kontext des Denkens von Hannah Arendt eine gewisse Antwort in der Analyse der Macht.

Dadurch, dass Arendt den öffentlichen Raum nicht als eine fixierte Größe ansieht, wird es möglich, dass sie das Schicksal des öffentlichen Raums als solche betrachten kann. Die Herausbildung und die Dynamik eines öffentlichen Raums sind für sie eng mit der Entstehung dessen verknüpft, was sie »Macht« nennt. Der als »Erscheinungsraum« verstandene öffentliche Bereich, in dem Menschen handelnd und sprechend miteinander umgehen, macht den Sinn der Polis und den häufigen Hinweis auf die Griechen in den Ausführungen von Arendt deutlich. Die Polis meint hier nämlich nicht die Stadt als geographisch lokalisierbaren Ort, sondern sie ist die »Organisationsstruktur« der Stadtbewohner, die sich nicht einfach, sondern »aus dem Miteinanderhandeln und -sprechen ergibt«, und zwar zwischen denen, die »um dieses Miteinander willen zusammenleben «. ${ }^{17}$ Damit wird auch der Sinn des öffentlichen Raums präzisiert, indem es nicht bloß darauf ankommt, dass Menschen voreinander erscheinen, wie andere Dinge der Welt, sondern »ausdrücklich in Erscheinung treten. ${ }^{18}$ Es handelt sich nicht mehr um eine »Öffentlichkeit« im Sinne von Zugänglichkeit für jedermann, es geht um ein ausdrückliches Erscheinen, und deswegen lässt sich die Herausbildung dieser Öffentlichkeit auch nicht als ein Ereignis auffassen, das automatisch aus dem Zusammenleben vieler folgt. Arendt zufolge ist es nun die Macht, die den Erscheinungsraum zwischen Handelnden und Sprechenden überhaupt ins Dasein ruft und der Erscheinungsraum lebt von dieser Macht. Die Macht ist demnach immer ein »Machtpotential «, das sich in einem menschlichen Miteinander bildet. ${ }^{19}$ Nimmt man den Hinweis auf die Organisationsstruktur einer Menschengruppe auf, wird auch verständlich, warum die Entstehung der Macht das menschliche Zusammensein voraussetzt: »Nur in einem Miteinander, das nahe genug ist, um

17 | Arendt: Vita activa, S. 250.

18 | Ebd.

19 | Aus dieser Bestimmung ist ersichtlich, dass die so verstandene Macht extrem unbestimmt ist, und das ist der Grund für die Rede vom Machtpotential, das nur in seinen Realisierungen zu erblicken ist, aber sich nie verdinglichen lässt: "Macht ist immer ein Machtpotential, und nicht etwas Unveränderliches, Meßbares, Verläßliches wie Kraft oder Stärke. Stärke ist, was ein jeder Mensch von Natur in gewissem Ausmaße besitzt und wirklich sein eigen nennen kann. Macht aber besitzt eigentlich niemand, sie entsteht zwischen Menschen, wenn sie zusammen handeln, und sie verschwindet, sobald sie sich wieder zerstreuen." Arendt: Vita activa, S. 252. 
die Möglichkeit des Handelns ständig offen zu halten, kann Macht entstehen [...] Was eine Gruppe von Menschen als Gruppe zusammenhält, wenn der immer flüchtige Augenblick des Zusammenhandelns verflogen ist, und was wir heute Organisation nennen, ist Macht, die wiederum ihrerseits dadurch intakt gehalten wird, daß die Gruppe sich nicht zerstreut. ${ }^{20}$ Derart hat Macht für Arendt im weitesten Sinne mit den gemeinsamen Handlungsmöglichkeiten einer Gruppe von Menschen zu tun, wobei es sich nicht einfach um den Vollzug gemeinsamer Handlungen handelt, da die Macht in den gemeinsamen Aktionen zwar zur Geltung kommt, aber sich nicht darin erschöpft. Demnach lässt sich Macht im Anschluss an Arendt als das inhaltlich noch unbestimmte Zusammenhalten einer Gruppe verstehen, das für sich genommen weder an bestimmte Ziele noch an bestimmte Organisationsformen gebunden ist. Die Rede von einer Gruppe meint also in diesem Zusammenhang nicht eine Menschengruppe überhaupt im Sinne eines bloßen Nebeneinanders von Menschen, sondern in einem noch zu klärenden Sinne »organisierte« oder »gegründete« Gruppen.

Die Macht bei Arendt hat zunächst nicht mit der Fähigkeit eines politischen Körpers, bestimmte Zwecke zu verfolgen, zu tun, sondern mit dem Handlungspotential von Gruppen, deren Mitglieder füreinander hinreichend ausdrücklich präsent sind und bleiben können. Demgegenüber wäre ein Bündnis zu einem bestimmten Zweck darin instrumentell, dass der Zweck des Zusammenseins von vornherein festgelegt wird. Ferner kann die Präsenz der Gruppenmitglieder füreinander nicht einfach ein physisches Zusammensein bedeuten, weil das allein gewiss nicht hinreichend dafür wäre, dass sie voreinander ausdrücklich erscheinen. Durch die Verhinderung des Zusammenseins lässt sich aber, wie Arendt es mit Blick auf totalitäre Regimes betont, auch die Entstehung von Macht unterbinden. Es handelt sich also darum, dass die Mitglieder der Gruppe aufeinander derart eingestellt sein müssen, dass das Miteinander-Reden und das gemeinsame Handeln möglich bleiben. Die Macht lässt sich demnach als jenes sich dynamisch ändernde, vorläufige Einverständnis bestimmen, das inhaltlich noch ganz unbestimmt ist und das die Mitglieder der Gruppe, sofern sie sich um öffentliche Angelegenheiten kümmern, aufeinander achten und sich aufeinander einstellen lässt. ${ }^{21}$ Sie ist weniger als eine konkrete Zielsetzung oder gemeinsame Handlung, weil sie nur die Bereitschaft zur gemeinsamen Diskussion und zum gemeinsamen Handeln bildet, und auch weniger als ein Konsens über bestimmte

20 | Ebd. S. 252ff.

21 Dieses Konzept bezieht sich auf solche, die zumindest bereit sind, politisch aktiv zu werden. Arendt ist sich natürlich dessen bewusst, dass nicht jeder sich um politische Gelegenheiten kümmert. Darin aber einen verwerflichen Elitarismus sehen zu wollen, wäre jedoch unbegründet: "Daß Arendts Denken einen gewissen Elitarismus in sich birgt, unterscheidet sie im Grunde nicht von anderen politischen Theoretikern. Keine politische Philosophie kommt ohne eine Unterscheidung zwischen politisch aktiven und passiven Bürgern aus." Schönherr-Mann: Hannah Arendt, S. 142. 
Überzeugungen oder Themen. Ein solches Einverständnis muss aber den öffentlichen Raum als Erscheinungsraum tragen, genauer gesagt »ins Dasein rufen«, weil ohne eine solche Bereitschaft und ohne vorläufiges Einverständnis Menschen nicht ausdrücklich voreinander erscheinen könnten. Andererseits kommt die so gedeutete Macht darin zur Geltung, dass der öffentliche Raum als solcher erhalten und beachtet bleibt. Die Frage, ob und wie eine Öffentlichkeit in diesem Sinne unter Bedingungen der heutigen Massengesellschaften, ja überhaupt unter Bedingungen der Moderne verwirklicht werden kann, bestimmt auch die Tragfähigkeit dieser Theorie der politischen Macht.

Diese eigentümliche Bestimmung der Macht bei Arendt wird dadurch für die politische Theorie relevant, dass sie einen wesentlichen Bestandteil eines jeden Gemeinwesens bildet: »Was einen politischen Körper zusammenhält, ist sein jeweiliges Machtpotential, und woran politische Gemeinschaften zugrunde gehen, ist Machtverlust und schließlich Ohnmacht. [...] und die Geschichte ist voll von Beispielen, die zeigen, daß kein materiell greifbarer Reichtum der Welt diesen Machtverlust auszugleichen vermag. Mit realisierter Macht haben wir es immer dann zu tun, wenn Worte und Taten untrennbar miteinander verflochten erscheinen, wo also Worte nicht leer und Taten nicht gewalttätig stumm sind «. ${ }^{22}$ Außerdem ist es nicht nur der politische Körper, dessen Kern die Macht konstituiert, sondern alle politischen Institutionen sind Arendt zufolge »Manifestationen und Materialisationen der Macht«, und dementsprechend erstarren und verfallen diese Institutionen, wenn »die lebendige Macht des Volkes« nicht mehr hinter ihnen steht. ${ }^{23}$ Auf Anhieb ist es gewiss nicht ganz klar, wie die lebendige Macht des Volkes zu verstehen sei. Die zitierte Stelle aus Macht und Gewalt gibt jedoch einen wichtigen Hinweis auf einen »ursprünglichen Konsens«, der die Institutionen und Gesetze erst ins Leben gerufen hat, und dessen Fortsetzung als Unterstützung des Volkes den politischen Institutionen und Gesetzen Macht verleiht. Um die Herausbildung dieses ursprünglichen Konsenses zu klären, muss man berücksichtigen, dass Arendt auch von einem »Machtursprung « spricht, der mit der Gründung der Gruppe zusammenfällt: »Macht bedarf keiner Rechtfertigung, da sie allen menschlichen Gemeinschaften immer schon inhärent ist. Hingegen bedarf sie der Legitimität. Macht entsteht, wann immer Menschen sich zusammentun und gemeinsam handeln, ihre Legitimität beruht nicht auf den Zielen und Zwecken, die eine Gruppe sich jeweils setzt; sie stammt aus dem Machtursprung, der mit der Gründung der Gruppe zusammenfällt. Ein Machtanspruch legitimiert sich durch Berufung auf die Vergangenheit, während die Rechtfertigung eines Mittels durch einen Zweck erfolgt, der in der Zukunft liegt. ${ }^{24}$

22 | Ebd., S. 252.

23 | Arendt: Macht und Gewalt, S. 42. Zum Problemkomplex der politischen Institutionen und deren Rolle im Denken Hannah Arendts siehe die sehr ausführliche Arbeit von Jürgen Förster.

24 | Ebd., S. 53. 
Dieser Hinweis spezifiziert die Gruppe, innerhalb der Macht entsteht: es ist nicht eine Gruppe gleichwelcher Art, sondern eine, die eigens gegründet werden musste. Bei der Frage, wie diese Gründung zu fassen ist, schwebt Arendt sicherlich die Gründung der Vereinigten Staaten, die constitutio libertatis, wie in einer Kapitelüberschrift des Buchs über die Revolution genannt wird, als Musterbeispiel vor Augen. Gleichwohl ist die Gründung der Vereinigten Staaten darin spezifisch, dass die Founding Fathers sich die Bewahrung der Freiheit als Ziel gesetzt haben. ${ }^{25}$ Die Entstehung der Macht im Sinne Arendts scheint aber nicht an ein bestimmtes Leitziel der Gruppe gebunden zu sein; es wäre sinnlos zu sagen, dass die Entstehung der Macht in der Gründung einer Gruppe notwendig auf die Bewahrung der Freiheit gerichtet sein muss. Dem entspricht, dass die Ausbildung einer dauerhaften Stätte der Freiheit nicht als »Macht«, sondern im Anschluss an den Sprachgebrauch von Condorcet als »Revolution« bezeichnet wird. Damit knüpft sich die Macht in Arendts Deutung an die Zusammensetzung einer Menschengruppe, ohne konkrete Ziele vorzuschreiben. Man könnte von einer eigentümlich modifizierten Vertragstheorie sprechen, ohne einen konkret beschriebenen Naturzustand, der die vom Vertrag zu realisierenden Ziele bestimmen würde.

Weder im Text von Vita activa noch in Macht und Gewalt gibt Arendt darüber Auskunft, wie sie die Gründung eines politischen Körpers im Einzelnen konzipiert. Das Essay »Ziviler Ungehorsam« skizziert eine »horizontale Version« des Gesellschaftsvertrags als die Form der Bildung politischer Gemeinwesen, und es stellt die Vorstellung von Arendt darüber dar, wie durch die Gründung einer Gruppe Macht entsteht. ${ }^{26}$ Unter der horizontalen Version versteht Arendt ein Gemeinwesen, das aus wechselseitiger Verpflichtung von verschiedenen Subjekten entsteht, und diese Art von Gründung wird von den Modellen des biblischen Bundes, der auf dem Gehorsam gegen Gott beruht, und der vertikalen Version des Gesellschaftsvertrags (Hobbes), in dem die Einzelnen ihr Gewaltpotential dem

25 | Helmut Dubiel macht in diesem Kontext auf die Doppeldeutigkeit des Begriffs "Konstitution" aufmerksam, die für unseren Zusammenhang aufschlussreich ist: Arendt bezieht den Begriff auf zwei getrennte Aspekte des Prozesses der Verfassungsgebung: "einmal auf sie als abgeschlossenen und dokumentarisch vorliegenden Akt, das andere Mal auf den Prozeß wechselseitiger Abstimmung, Beratung und Debatte, der diesem Akt vorausging. Erst Hannah Arendts Theorie der Macht erlaubt es uns zu begreifen, daß dieser Prozeß der Instituierung einer Verfassung, die sie am Fall der amerikanischen Verfassung nachgezeichnet hat, mindestens so wichtig ist wie das Dokument, in dem sich ihr Abschluß niederschlägt. Die politische Autorität dieses Dokuments, die sich z.B. in der Schlichtung zukünftiger Konflikte zu bewähren hat, kann nur so groß sein wie die kommunikative Energie, die während des Beratungsprozesses in sie eingegangen ist." Dubiel: Das nicht angetretene Erbe, S. 49.

26 | Vgl. dazu Schindler: Geglückte Zeit, S. 257. 
Souverän zuschreiben, unterschieden. ${ }^{27}$ Dabei besteht die Funktion der wechselseitigen Verpflichtung darin, die Zukunft in »menschenmöglichem Ausmaß berechenbar und verläßlich zu machen «. ${ }^{28}$ Gleichwohl ist es nicht ganz eindeutig, dass sich Arendt die horizontale Version des Gesellschaftsvertrags zu eigen macht. Der Aufsatz »Ziviler Ungehorsam« behandelt ja den konkreten Fall der Vereinigten Staaten, und allein schon deswegen scheint es fraglich, ob die geschilderte Form des Gesellschaftsvertrags auch das Fundament ihres Machtbegriffs abgeben sollte. Ein wesentlicher Unterschied von Arendts Konzeption und der Vertragstheorie besteht darüber hinaus darin, dass es für Arendt kein konkretes Ziel des Vertrags gibt.

Von diesen Aspekten her lassen sich wichtige Bestimmungen des Machtbegriffs von Hannah Arendt besser verstehen. Zum einen wird daraus klar, dass die Macht ihrem Wesen nach schrankenlos ist, woraus folgt, dass Macht nur durch die gleichzeitige Existenz von anderen Machtgruppen, also durch Pluralität begrenzt werden kann. ${ }^{29}$ Zum anderen resultiert ein sehr charakteristischer Zug der Machtauffassung Arendts, die scharfe Entgegensetzung von Macht und Gewalt aus den ausgeführten Überlegungen. Die Macht wird von der Gewalt und jeder Art von Zwang abgehoben, und damit wird die Gewalt vom Zwangscharakter her gedeutet. Derart wird Gewalt abhängig von Gewaltmitteln, wogegen Macht wesentlich unabhängig von materiellen Faktoren ist, wenngleich sie als einzige materielle Voraussetzung vom Zusammensein von Menschen abhängig ist. Obwohl sich dieses Zusammensein mit Gewalt verhindern lässt, kann die Gewalt derart die Macht nur zerstören, aber nicht ersetzen. ${ }^{30}$ Schließlich folgt aus der gegebenen Bestimmung der Macht für Arendt, dass keiner die Macht »besitzen« kann, sondern eine solche Redeweise nur so viel besagen kann, dass einer von mehreren beauftragt wird, in ihrem Namen zu handeln.

\section{NARRATIVITÄT}

Bisher wurde in groben Zügen dargestellt, wie Hannah Arendt das Politische mit der Öffentlichkeit eng verknüpft. Es wurde bereits auch ihre Überzeugung berührt, der zufolge die Einzigartigkeit und Einmaligkeit von Individuen sich nur

27 | Arendt: Ziviler Ungehorsam, S. 145. "Die Figur der , Gründung، ist hier mißverständlich, handelt es sich doch um einen Vertrag zwischen einer schon bestehenden Gesellschaft und einem außerhalb ihres Kontextes lokalisierten Herrscher. Der hypothetische Urvertrag hat einzig die Funktion, eine bereits ausgeübte Herrschaft zu rechtfertigen." Dubiel: Das nicht angetretene Erbe, S. 45.

28 | Arendt: Ziviler Ungehorsam, S. 151.

29 | Arendt: Vita activa, S. 254.

30 | Ebd., S. 255. 
in einer Öffentlichkeit, nur vor einem Gegenüber zeigen kann. ${ }^{31}$ Die politische Theorie von Arendt wurde auch mehrfach wegen des vermeintlichen Übergewichtes der Öffentlichkeit kritisiert, mit der Begründung etwa, sie stelle die Politik als Spielplatz vor, wo Egomanen sogenannte "große Taten« vollziehen wollen. Sicherlich ist das Konzept des Politischen bei der deutschen Philosophin in gewissem Sinne ambivalent: an einigen Stellen legt sie nahe, das Politische habe mit Herausbildung, Erhaltung und Verschwinden, kurz: mit der Dynamik der Macht zu tun, an anderen Stellen aber wird der öffentliche Raum als Ort beschrieben, wo sich die Vortrefflichkeit und mithin die Einzigartigkeit der Einzelnen zeigen kann. Die beiden Aspekte jedoch - Dynamik der Macht und dauerhafte Einzigartigkeit - sind keineswegs ohne weiteres identisch; es ist sogar möglich, dass sie miteinander nur unter bestimmten Voraussetzung zu tun haben. Dass es sich um unterschiedliche Phänomene handelt, zeigt eine Passage aus Vita activa, wo Arendt sagt, die Griechen haben die Mühe des Gemeinwohls nur auf sich genommen, um sich in ihrer Einzigartigkeit und Vortrefflichkeit zeigen zu können. ${ }^{32}$ Jedenfalls braucht sowohl die Vortrefflichkeit wie auch die negative Einzigartigkeit (Schlechtigkeit) ein »Publikum«, und deswegen ist die Verbindung zwischen der Pluralität und dem Politischen ohne den Gedanken einer starken Individualität undenkbar. Man sollte jedoch zwischen der Erscheinung oder Erfassung der Einzigartigkeit und deren Rolle in der Pluralität und im Konzept des Politischen unterscheiden. Der Aspekt der Narrativität, den es jetzt zu erörtern gilt, scheint auf den ersten Blick den ersten Gesichtspunkt zu betreffen. Bei näherer Betrachtung stellt sich heraus, dass nach Arendt auch die großen Taten eine narrative Struktur haben bzw. haben müssen, die eine Öffentlichkeit in eine politische verwandeln lassen. Arendt verbindet auf explizite Weise die Öffentlichkeit und die großen, bedeutenden Taten, genauer gesagt: in Verbindung gebracht wird das Politisch-Werden eines öffentlichen Raums und die als organisiertes Gedächtnis aufgefasste Öffentlichkeit, die polis. Dazu heißt es in einem aufschlussreichen Fragment aus Was ist Politik?:

31 Auf bemerkenswerte Weise bleibt es in diesem Zusammenhang unklar, ob für Arendt Gott so ein Gegenüber sein kann. Eine verneinende Antwort scheint daraus zu folgen, wie sie den Öffentlichkeitsverlust durch die frühchristliche und mittelalterliche, vom Christentum motivierte Abwendung vom öffentlichen HandeIn beschreibt.

32 | Die als öffentlicher Raum verstandene Polis betrachtet Arendt als Ort des Wettstreits in der Vortrefflichkeit, "in dem ein jeder sich dauernd vor allen anderen auszeichnen mußte [...] Mit anderen Worten, der öffentliche Raum war gerade dem Nicht-Durchschnittlichen vorbehalten, in ihm sollte ein jeder zeigen können, wodurch er über das Durchschnittliche hinausragte. Um dieser Möglichkeit willen, das Außerordentliche zu leisten und geleistet zu sehen, waren die Bürger der Polis dann auch mehr oder weniger bereit, ihren Teil an der Rechtsprechung, der Verteidigung, der Verwaltung der Stadt auf sich zu nehmen " Arendt: Vita activa, S. 53. 
"[I]n der Welt, die sich den Beherzten, den Abenteurern und Unternehmungslustigen öffnet, [entsteht] zwar bereits eine Art öffentlicher, aber noch nicht ein im eigentlichen Sinne politischer Raum. Öffentlich wird dieser Bereich, in den die Tatenlustigen vorstoßen, weil sie unter ihresgleichen sind und einander jenes Sehen und Hören und Bewundern der Taten gewähren können, auf deren Hörensagen hin der Dichter und Geschichten-Erzähler dann später innen den Ruhm bei der Nachwelt sichern kann. Im Gegensatz zu dem, was im Privaten und in der Familie geschieht, in der Verborgenheit der eigenen vier Wände, erscheint hier alles in jenem Licht, das nur in die Öffentlichkeit, und das heißt die Anwesenheit der Anderen, erzeugen kann. Aber dies Licht, das die Vorbedingung allen wirklichen Erscheinens ist, ist trügerisch, solange es nur öffentlich und nicht politisch ist. Der öffentliche Raum des Abenteuers und des Unternehmens verschwindet, sobald alles an sein Ende gekommen ist, das Heereslager aufgelöst ist und die 'Helden ‘ [...] wieder nach Hause zurückgekehrt sind. Politisch wird dieser öffentliche Raum erst, wenn er in einer Stadt gesichert ist, also an einen greifbaren Platz gebunden, der sowohl die denkwürdigen Taten wie die Namen der denkwürdigen Täter überleben und der Nachwelt in der Folge der Geschlechter überliefern kann. Diese Stadt, die den sterblichen Menschen und ihren flüchtigen Taten und Worten eine bleibende Stätte bietet, ist die Polis“. ${ }^{33}$

Der Zusammenhang besteht also Arendt zufolge zwischen dem öffentlichen Raum und den bedeutsamen Taten, sofern sie in erzählter Form bewahrt und erinnert werden. Der öffentliche Raum wird demnach für Arendt nicht allein schon deshalb ein politischer, weil er alles, was in ihn eintritt, zugänglich macht. Gleichwohl handelt es sich um zwei Aspekte, die das Politisch-Werden einer Öffentlichkeit bedingen: zum einen soll der öffentliche Raum ein konkreter und als solcher derart dauerhaft sein, dass er nicht sofort mit dem Abenteuer und den Handlungen verschwindet, zum anderen kommt es auf die Bewahrung konkreter Handlungen als beispielhaft für eine politische Gemeinschaft an. Im Hinblick auf den ersten Aspekt wurde der öffentliche Charakter bereits skizzenhaft geklärt, während mit Blick auf den zweiten die Mechanismen der Bewahrung durch Narrativen noch erläutert werden sollen. Gezeigt werden soll im Folgenden, wie eingangs schon bemerkt, dass es Arendt aus strukturellen Gründen nicht darauf ankommt, die Zugänglichkeit eines jeden zur eigenen Identität zu klären, vielmehr darauf, wie beispielhafte Taten und Leben innerhalb eines öffentlichen Raumes aufbewahrt und erinnert werden können.

Zu diesem Zweck sollte zunächst der Handlungsbegriff bei Arendt näher bestimmt werden. Hannah Arendt nimmt eine Dreiteilung menschlicher Tätigkeiten vor, die dadurch die Grundlage für ihre Diskussion des Politischen bildet, dass sie die politische Tätigkeit als Handeln im eigentümlichen Sinne begreift. Arendts Differenzierung menschlicher Aktivitäten in Arbeit, Herstellen und Handeln lässt sich programmatisch als eine scharfe Kritik an der philosophischen Überlieferung verstehen: die Philosophie ist prinzipiell für die theoretische

33 | Arendt: Was ist Politik, S. $45 f$. 
Abwertung und Zurückdrängung des öffentlich-politisch-praktischen Lebens verantwortlich. ${ }^{34}$ Das aus dieser Diagnose sich ergebende Programm kann als Rehabilitierung der vita activa gefasst werden und steht im Zentrum des philosophischen Hauptwerkes Vita activa oder Vom tätigen Leben. Die drei Tätigkeitstypen - Arbeit, Herstellen und Handeln - unterscheiden sich nicht nur voneinander, sondern sie entsprechen darüber hinaus drei grundsätzlichen Bedingungen menschlichen Lebens. Als Arbeit definiert Arendt jede Tätigkeit, die für die Erhaltung des Lebensprozesses des Einzelnen verantwortlich ist, und darin weist die Arbeit auf das Leben hin. Herstellen dagegen bezeichnet jene Tätigkeiten, die eine dauerhafte Welt der Gegenstände, die Menschen ein Heim bieten kann, also die Weltlichkeit im Sinne Arendts hervorbringen. Schließlich fasst Arendt das Handeln als die Enthüllung der Einzigartigkeit des Handelnden, und auf diese Weise entspricht das Handeln der Pluralität, die mit dem Erscheinen und zugleich mit dem Erfassen des Handelnden zu tun hat. ${ }^{35}$

Diese Typologie menschlicher Tätigkeiten wurde in der Literatur über Arendt oft kritisiert. ${ }^{36}$ Sowohl bei der Unterscheidung von Arbeit und Herstellen wie auch bei der von Herstellen und Handeln ist es problematisch, dass sie sich nicht allein aufgrund einer Beschreibung der Tätigkeiten deutlich machen lässt. ${ }^{37} \mathrm{Wie}$ anderswo gezeigt wurde, kann man jedoch in beiden Fällen darauf bestehen, dass die Unterscheidung aus der Perspektive des Handelnden sinnvoll bleibt. ${ }^{38}$ Selbst wenn aus der Beobachtungsperspektive nicht eindeutig festzustellen ist, ob jemand als Hobby oder zum Geldverdienen (also als Arbeit) Holz fällt, ist es aus der

34 | An einigen Stellen scheinen auch andere Faktoren, wie etwa das Christentum mit seiner spezifischen Vermeidung der Öffentlichkeit, eine Rolle zu spielen. Für eine eingehende Erörterung des komplexen Verhältnisses von Arendt zur öffentlichen Religion vgl. den Aufsatz von Gángó: Hannah Arendt.

35 | Arendt: Vita activa, S. $16 \mathrm{ff}$.

36 | "Attempts to distinguish among types of human activity, such as labor, work, and action in Arendt's case ... are subject to a standard objection: it is pointed out that any complex human activity, form factory work, to writing a book, to making a meal, cannot simply be seen as an exemplar of a single action type. ... When human acitivities are considered as complex social relations, and contextualized properly, what appears to be one type of activity may turn out to be another; or the same activity may instantiate more than one action type." Benhabib: The Reluctant Modernism, S. 131. Siehe z.B. das Buch von Trottmann: Faire.

37 | Eine ähnliche Schwierigkeit ergibt sich mit Blick auf das Denken. Arendt stellt den Denkprozess dem sprachlich formulierten Gedanken (z.B. dem geschriebenen Aufsatz) mit der Begründung gegenüber, dass Letztere bereits zur Kategorie des Herstellens gehört. Diese Beschreibung verkennt m.E., dass das Denken nicht ohne die Formulierung von einzelnen Gedanken auskommt, und derart gehört das "reine" Denken mit einzelnen Gedanken als seinen Resultaten zusammen.

38 | Olay: Cura rei publicae. 
Perspektive des Handelnden ganz unproblematisch zu entscheiden, um welchen Fall es geht. Selbst wenn die Unterscheidung von Arbeit und Herstellen problematisch sein sollte, folgt daraus für sich genommen noch nichts für die Deutung der politischen Tätigkeit als Handeln.

Was den Gebrauch des Ausdrucks »Handeln« bei Arendt betrifft, ist er darin ganz unüblich enger als der alltägliche Gebrauch, weil im Deutschen der Ausdruck jede Tätigkeit überhaupt bezeichnen kann. Die Eigentümlichkeit ihrer Auffassung lässt sich am besten in Abhebung vom Konzept des Aristoteles verdeutlichen. Nach Aristoteles liegt das Unterscheidungsmerkmal von Herstellen und Handeln (poiesis und praxis) im Verhältnis vom Ziel der Tätigkeit und der Tätigkeit selbst. Während das Ziel der Tätigkeit im Herstellen außerhalb desselben liegt, liegt das Ziel im Handeln innerhalb des Vollzugs. Arendt folgt nun Aristoteles darin, dass die Arbeit und das Herstellen ihr Ziel außerhalb von sich haben, wogegen das Handeln sein Ziel in sich trägt. Ihr Handlungsbegriff wird aber enger gefasst, da bei Aristoteles auch solche Tätigkeiten zur Praxis gehören (etwa das Spiel und die Unterhaltung), die im Sinne Arendts keine Handlungen sind, weil sie den Handelnden in seiner Einzigartigkeit nicht enthüllen. Der Grund dieser Abweichung besteht darin, dass Arendt Handlung nicht über das Verhältnis der Tätigkeit zu deren Ziel definiert, sondern dadurch, dass der Handelnde sich in ihr offenbart: im Handeln »zeigt sich« der Handelnde als dieser Einzelne im Gegensatz zu anderen. ${ }^{39}$

Für Hannah Arendt sind Sprechen und Handeln die Tätigkeiten, in denen die Einzigartigkeit des eines Menschen sich zeigt, wobei die Rede von Einzigartigkeit anstelle der Verschiedenheit den Umstand unterstreichen soll, dass es um ein aktives Sich-Unterscheiden und nicht um ein bloßes Verschieden-Sein geht. ${ }^{40}$ Der Gedanke wird etwas später in einer wesentlichen Hinsicht präzisiert, weil sich vor allem durch Reden zeigen kann, wer der Handelnde ist. Zwar besteht die enge Verbindung von Reden und Handeln darin, dass beide auf die Frage »Wer bist Du?« antworten, aber gleichzeitig räumt Hannah Arendt den Reden eine gewisse Priorität ein. ${ }^{41}$ Das bedeutet freilich nicht, dass das einmalige »Wer-einer-ist « eindeutig sprachlich formuliert werden könnte, wofür Arendt als letzten Grund den Unterschied zwischen Personen und verfügbaren Sachen angibt: »das unverwechselbar einmalige des Wer-einer-ist« eines jeden, »das sich so handgreiflich im Sprechen und Handeln manifestiert, entzieht sich jedem Versuch, es eindeutig in Worte zu fassen. Sobald wir versuchen zu sagen, wer jemand ist, beginnen wir Eigenschaften zu beschreiben, die dieser Jemand mit anderen teilt und die

39 | Arendt betont auch, dass Handeln im Sinne des Neuanfangens auch mit der Freiheit zusammenhängt: "Die Tatsache, daß der Mensch zum Handeln im Sinne des Neuanfangens begabt ist, kann [...] nur heißen, dass er sich aller Absehbarkeit und Berechenbarkeit entzieht" Arendt: Vita activa, S. 217.

$40 \mid$ Ebd., S. 214.

41 | Ebd., S. $217 f$. 
ihm gerade nicht in seiner Einmaligkeit zugehören. Es stellt sich heraus, daß die Sprache, wenn wir sie als Mittel der Beschreibung des Wer benutzen wollen, sich versagt und an dem Was hängen bleibt«. ${ }^{42}$ Damit stellt sich die eigentümliche Schwierigkeit, dies Wer-einer-ist, »das eigentlich Personale« zugänglich zu machen. Ferner denkt Arendt dies Wer-einer-ist auf eine zutiefst anti-kartesianische Weise so, dass es der jeweiligen Person nicht von vornherein zugänglich ist, sondern diese Person im Sprechen und Handeln auch für sie selbst erst enthüllt wird. $^{43}$

Zunächst sollte festgestellt werden, dass es wesentlich zum Handlungsbegriff von Hannah Arendt - und, wie ergänzt werden muss, zum Begriff der Reden gehört, dass sie auf den öffentlichen Raum, der durch die Anwesenheit anderer konstituiert wird, angewiesen sind. Die Pluralität ist in diesem Zusammenhang auf doppelte Weise Vorbedingung des Handelns. Zum einen ist sie die Bedingung dafür, dass etwas sich zeigen kann, die Pluralität, wie Arendt sie fasst, die radikale Einzigartigkeit des einzelnen Menschen impliziert. ${ }^{44}$ Zum anderen bildet sie das »Medium«, worin der Handelnde sich zeigen kann, denn die Pluralität in diesem Sinne ist der Erscheinungsraum, wo Handlungen vollzogen werden können, derart, dass sie Realität gewinnen, indem andere sie sehen und über sie berichten können. Der öffentliche Raum sichert also einen Ort, wo Handlungen, die sonst mit dem Ende ihres Vollzugs verschwinden würden, eine dauerhafte Existenz erhalten können. ${ }^{45}$ Eine narrative Festlegung, also die Formulierung in einer Erzählung braucht das Handeln also deswegen, weil es von sich aus kein Resultat erzeugt, das es überdauern würde: »Das Handeln ist die einzige Tätigkeit der Vita activa, die sich ohne Vermittlung von Materie, Material und Dingen direkt zwischen Menschen abspielt «. ${ }^{46}$ Diese Überlegung führt aber aufgrund der

42 | Ebd., S. $222 f$.

43 | "The fundamental gain derived from action, though not its goal, is self-knowledge. According to Arendt, we do not know ourselves and than reveal to others what we know. Rather, we discover who we are in the process of revealing ourselves to others. "Zaretsky: Hannah Arendt, S. 223.

44 | Die "menschliche Pluralität ist eine Vielheit, die die paradoxe Eigenschaft hat, daß jedes ihrer Glieder in seiner Art einzigartig ist." ebd., S. 214.

45 | Diese beiden grundlegenden Aspekte zeigen deutlich, dass die politische Ausarbeitung der Pluralität zum Zentrum des Denkens von Hannah Arendt gehört. "Human togetherness implies a world that exists between them, in which we all appear, in which we all perceive one another. This 'world، is equally lost through rulership-based political theories as well as through liberalism's individualistic-based political expression. Neither recognizes the importance and necessity of both distinctness and interaction. In fact, Bernstein claims that, from the perspective of Arendtian political philosophy, liberalism's understanding of plurality can only appear superficial."Hull: The Hidden Philosophy, S. 22.

46 | Arendt: Vita activa, S. 17. 
Voraussetzungen von Arendt zu der unvermeidlichen Leistung des Erzählers, der die Geschichte der Taten in Gestalt einer überlieferbaren Geschichte formuliert.

Eine dieser Voraussetzungen wurde bereits oben berührt: Arendt geht es um die Wirklichkeit von Taten und exemplarischen Leben in und für eine Öffentlichkeit, die diese Taten und diese Lebensabläufe überdauert. Ihre mögliche Bedeutung und Wirkung hängt dabei offenbar davon ab, ob sie in tradierbarer Form weitergegeben werden können oder nicht. Damit handelt es sich offensichtlich nicht oder zumindest nicht allein um die Frage, wie jemand die eigene Einzigartigkeit und Identität mithilfe einer Geschichte fassen kann. Es geht mit anderen Worten nicht um die eigene Lebensgeschichte, sondern um eine Lebensgeschichte, die anderen zugänglich gemacht werden kann. Es leuchtet ein, dass eine solche Geschichte nur von einem Beobachter erzählt werden kann. Arendt argumentiert zwar dafür, dass eine Handlung und eine Lebensgeschichte nur von einem Beobachter erzählt werden kann, aber ihre Argumente können das nur unter ihren spezifischen Voraussetzungen einer politischen Öffentlichkeit plausibel machen. Die Form der Erzählung macht zwar eine wesentliche Gemeinsamkeit mit den narrativen Theorien der personalen Identität aus, aber die Unverzichtbarkeit des Beobachters folgt allein aus dem spezifischen Interesse Arendts. An dieser Stelle lässt sich ein wesentlicher Unterschied zwischen dem Identitätskonzept von Arendt und dem von Theoretikern einer narrativen persönlichen Identität, etwa von McIntyre oder Ricœur, angeben, da diese Autoren im Gegensatz zu Arendt vom Vorrang der Perspektive der ersten Person ausgehen, indem sie die Frage stellen, wie einem die eigene Identität zugänglich wird. Wenn Ricœur in der Frage nach dem »Wer« bei Arendt eine Vorläuferin seiner Fragestellung zu erkennen glaubt, übersieht er die Fragerichtung bei Arendt, die nicht auf die Erfassung der personalen Identität der jeweiligen Person zielt, sondern auf das dauerhafte Zugänglich-Machen einzelner Taten und exemplarischer Leben.$^{47}$ Diese Präzisierung erklärt auch, warum für Ricœur die Beobachtungsperspektive keine Rolle spielt. Das bedeutet nicht, dass Arendts Überlegungen von vornherein nicht für

47 | "Le rejeton fragile issu de l'union de l'histoire et de la fiction, c'est l'assignation à un individu ou à une communauté d'une identité spécifique qu'on peut appeler leur identité narrative. , Identité , est pris ici au sens d'une catégorie de la pratique. Dire l'identité d'un individu ou d'une communauté, c'est répondre à la question : qui a fait telle action ? qui en est l'agent, l'auteur ? II est d'abord répondu à cette question en nommant quelqu'un, c'està-dire en le désignant par un nom propre. Mais quel est le support de la permanence du nom propre ? Qu'est-ce qui justifie qu'on tienne le sujet de l'action, ainsi désigné par son nom, pour le même tout au long d'une vie qui s'étire de la naissance à la mort ? La réponse ne peut être que narrative. Répondre à la question , qui ? ', comme l'avait fortement dit Hannah Arendt, c'est raconter l'histoire d'une vie. L'histoire racontée dit le qui de l'action. L'identité de qui n'est donc elle-même qu'une identité narrative." Ricœur: Temps et récit, S. $354 f$. 
eine Theorie der narrativen Identität lehrreich sein könnten, aber ihre Gedanken sollten nicht auf dieses Grundmotiv oder Leitziel hin geprüft werden. ${ }^{48}$

Arendt ist sich dieser impliziten Vorentscheidung für die Perspektive des Beobachters nicht bewusst und versucht zu begründen, warum eine Handlung und auch eine Lebensgeschichte nur aus der Beobachterperspektive beschrieben werden kann. Der wohl wichtigste Schritt ihrer Argumentation betrifft das »Bezugsgewebe menschlicher Angelegenheiten«, das wie folgt eingeführt wird:

[D]iese unwillkürlich-zusätzliche Enthüllung des Wer des Handelns und Sprechens [bildet] einen so integralen Bestandteil allen, auch des robjektivsten`, Miteinanderseins, daß es ist, als sei der objektive Zwischenraum in allem Miteinander, mitsamt der inm inhärenten Interessen gleichsam, von einem ganz und gar verschiedenen Zwischen durchwachsen und überwuchert [...]. Dieses zweite Zwischen, das sich im Zwischenraum der Welt bildet, ist ungreifbar, da es nicht aus Dinghaftem besteht und sich in keiner Weise verdinglichen oder objektivieren läßt; Handeln und Sprechen sind Vorgänge, die von sich aus keine greifbaren Resultate und Endprodukte hinterlassen. Aber dies Zwischen ist in seiner Ungreifbarkeit nicht weniger wirklich als die Dingwelt unserer sichtbaren Umgebung. Wir nennen diese Wirklichkeit das Bezugsgewebe menschlicher Angelegenheiten, wobei die Metapher des Gewebes versucht, der physischen Ungreifbarkeit des Phänomens gerecht zu werden. ${ }^{49}$

Das Bezugsgewebe bedeutet im Hinblick auf die Handlung, dass sie für den Handelnden eine Unberechenbarkeit mit sich bringt, weil es verhindert, dass der Handelnde seine Ziele wie geplant realisiert. Deswegen kommt Arendt zur interessanten Schlussfolgerung, dass das wahre Ergebnis des Handelns nicht die Realisierung vorher geplanter Ziele und Zwecke, sondern »die von ihm ursprünglich gar nicht intendierten Geschichten « ist, »die sich ergeben, wenn bestimmte Ziele verfolgt werden, und die sich für den Handelnden selbst erst einmal wie nebensächliche Nebenprodukte seines Tuns darstellen mögen. Das, was von seinem Handeln schließlich in der Welt verbleibt, sind nicht Impulse, die ihn selbst in Be-

48 | Ein Generalverdacht gegen die Idee der Lebensgeschichte überhaupt wurde von Pierre Bourdieu formuliert, der über "l'illusion biographique" und über "l'idéologie de sa propre vie " spricht: "parler d'histoire de vie, c'est présupposer au moins, et ce n'est pas rien, que la vie est une histoire et qu'une vie est inséparablement l'ensemble des événements d'une existence individuelle conçue comme une histoire et le récit de cette histoire" Bourdieu: L'illusion biographique, S. 81. Interessanterweise erwägt Arendt in einer Passage von Vita activa eine ähnlich akzentuierte "Verlegenheit", die daher kommt, dass "jede Abfolge von Geschehnissen, wenn sie nur zeitlich verbunden ist und gleich, wie zufällig und disparat die Veranlassungen jeweils sein mögen, immer noch genug Zusammenhang aufweist, um erzählbar zu sein und in dem Erzähltwerden einen Sinnzusammenhang zu ergeben." Arendt: Vita activa, S. 229. Der Einwand von Bourdieu bleibt in dem Sinne zu abstrakt, als es unklar bleibt, worin sein Gegenvorschlag bestünde.

49 | Ebd., S. 225. 
wegung setzten, sondern die Geschichten, die er verursachte; nur diese können am Ende [...] im Gedächtnis der Generationen wieder und wieder nacherzählt und in allen möglichen Materialien vergegenständlicht werden. ${ }^{50}$

Die Geschichten, von denen Arendt spricht, sind noch sprachlich zu formulieren oder anderswie zu vergegenständlichen. Sie sind also res gestae, und noch nicht historia rerum gestarum. An dieser Stelle führt Arendt die Leistung des Erzählers ein, die wegen der strukturellen Beschränktheit der Perspektive des Handelnden notwendig ist. ${ }^{51}$ Die Aktivität des Verfassers bleibt aber dabei auf eine merkwürdige Weise unterbetont: »Obwohl [...] erzählbare Geschichten die eigentlichen »Produkte« des Handelns und Sprechens sind, [...] mangelt der Geschichte selbst gleichsam ihr Verfasser. Jemand hat sie begonnen, hat sie handelnd dargestellt und erlitten, aber niemand hat sie ersonnen. ${ }^{52}$ Damit ist zwar zunächst gemeint, dass eine solche Geschichte nicht bewusst gelenkt oder erfunden wird. Man kann die These auch so verstehen, dass die erzählte Geschichte einer Handlung oder eines Lebens nicht konstruktivistisch verstanden werden sollte. Trotzdem scheint diese Überlegung die Möglichkeiten der Gestaltungsfreiheit im Erzählen eines jeden Geschehens nicht hinreichend zu berücksichtigen. Ein weiteres Problem besteht darin, dass das Sich-Zeigen des Handelnden in seinen Taten sich nur mit Einschränkungen geltend machen lässt. Der Handelnde enthüllt sich ja nicht als sich selbst, sondern als Täter dieser bestimmten Taten, welche Taten sich ihrer Einzigartigkeit zum Trotz mit anderen Taten vergleichen lassen, und so bewegen sie sich auf der Ebene einer gewissen Allgemeinheit. Derart können diese Taten die unverwechselbare Einzigartigkeit, die strenge Individualität des Handelnden nicht ausdrücken. Arendt könnte jedenfalls darauf entgegnen, dass sie nicht anzunehmen braucht, der Handelnde sei imstande, sich vollkommen und restlos in seinen Taten zu enthüllen, und auch mit dieser Einschränkung kann die These, dass der Handelnde in seiner Einzigartigkeit nur in seinen Handlungen zeigen kann, aufgestellt werden.

Ziehen wir Fazit: Arendts Betonung der Notwendigkeit des Beobachters und der Unmöglichkeit der Erzählung der eigenen Identität schien nicht überzeugend, weil man geneigt ist zu sagen, dass man das intimste Verhältnis zu dem hat, wer man ist. Nimmt man die Auffassung von Hannah Arendt ernst, folgt daraus, dass wir ohne eine überzeugende Vorstellung davon, wer wir sind, leben und das ist sicherlich unplausibel. Ihr zufolge weiß ich prinzipiell bis zu meinem Tod nicht, wer ich bin, und erst danach wird ein Beobachter imstande sein, meine Lebensgeschichte zu erzählen. Darauf könnte jedoch geantwortet werden, dass man grundsätzlich immer seine Lebensgeschichte als Antwort auf die Frage, wer

50 | Ebd., S. $226 f$.

51 | Die "volle Bedeutung dessen, was sich handelnd jeweils ereignete, [kennen] nicht diejenigen, die in das Handeln verstrickt waren und direkt von ihm betroffen, sondern derjenige, der schließlich die Geschichte überblickt und sie erzählt." ebd., S. 240.

52 | Ebd., S. 227. 
er oder sie ist, zu erzählen fähig ist. Ferner gründet man in der Regel das eigene Leben in einem elementaren Sinne auf diese Lebensgeschichte, die die persönliche Identität umschreibt und Handlungen, Entscheidungen und Bewertungen mitbestimmt. Es scheint übertrieben, dass aus der Perspektive Arendts all das nur Selbstverkennen und falsches Selbstbild sein könnte. Selbstverständlich ist es möglich, dass ich mich mit Bezug auf mich selbst in mehrerer Hinsicht täusche, irre, möglicherweise kann ich mich selbst bewusst oder unbewusst stilisieren usw., aber all diese Möglichkeiten könnten noch in einer Auffassung integriert werden, die ein narrativ strukturiertes Erfassen der eigenen Identität zumindest denken lässt. Natürlich hat Arendt Recht, als sie das Moment des Mitgenommenseins in einer Situation betont. Sie berücksichtigt jedoch nicht die Möglichkeit der späteren Reflexion, wo es doch im Prinzip möglich ist, sich von dem Geschehen zu distanzieren und dadurch von sich ein Bild zu machen, und zwar unabhängig davon, ob andere dieses Bild für überzeugend halten oder nicht. In diesem Sinne geht die These der Selbstignoranz bei Hannah Arendt, die erst aus einer nachträglichen Beobachtungsperspektive aufgeklärt werden könnte, zu weit.

Die Eigentümlichkeit von Arendts Gedankengang kommt aus ihrer eigentümlichen Perspektive, die in erster Linie auf die Nachwirkung von großen Taten und Leben auf eine Öffentlichkeit fokussiert. Dies Interesse macht auch den Akzent, der auf die Beobachterperspektive gelegt wurde, verständlich. Damit zeigt sich Narrativität für Arendt als Grundstruktur der wesentlichen Elemente einer politischen Öffentlichkeit, als offene Menge von beispielhaften Leben und Taten. Mit anderen Worten, Arendt fragt nicht danach, wie einer die eigene Identität zu fassen imstande ist; sie fragt vielmehr, wie vergangene große Taten und Personen als erzählbare Maßstäbe und Motivationen für alle, die am organisierten Gedächtnis der konkreten Öffentlichkeit teilnehmen, weitergegeben werden können. Mit dieser Präzisierung ihrer Perspektive lässt sich vielleicht die mehrmals bemerkte Zweideutigkeit des Handlungsbegriffs bei Arendt erläutern: Handeln als agonales Hervortretenlassen der Individualität und Handeln als machterzeugendes Miteinanderhandeln bilden weniger einen Widerspruch innerhalb eines als einheitlich geplanten Handlungsbegriffs, sondern zwei Aspekte der Leistungen des Handelns im Zusammenhang des Politischen.

\section{LITERATUR}

Arendt, Hannah: Ich will verstehen, München/Zürich o.J.

- Macht und Gewalt, München 1975.

- Vita activa, München/Zürich 2002.

— Was ist Politik?, München/Zürich 2003.

— »Ziviler Ungehorsam«, in: dies., Zur Zeit: politische Essays, Hamburg 1999, S. 119-159.

Benhabib, Seyla: The Reluctant Modernism of Hannah Arendt, London 1996. 
Bourdieu, Pierre: »L'illusion biographique«, in: P. Bourdieu: Raisons pratiques.

Sur la théorie de l'action, Paris 1994, S. 81-89.

Breier, Karl-Heinz: Hannah Arendt, Hamburg 2001.

Dubiel, Helmut: »Das nicht angetretene Erbe. Anmerkungen zu Hannah Arendts politischer Theorie«, in: H. Dubiel, Ungewißheit und Politik, Frankfurt a.M. 1994 , S. 29-66.

Förster, Jürgen: Die Sorge um die Welt und die Freiheit des Handelns: zur institutionellen Verfassung der Freiheit im politischen Denken Hannah Arendts, Würzburg 2009 .

Gángó, Gábor: »Hannah Arendt and the Problem of Public Religion«, in: P. Losonczi/A. Singh (Hg.), From Political Theory to Political Theology. Religious Challenges and the Prospects of Democracy, London/New York 2010, S. 47-59. Höffe, Ottfried: »Politische Ethik im Gespräch mit Hannah Arendt«, in: P. Kemper (Hg.), Die Zukunft des Politischen. Ausblicke auf Hannah Arendt. Frankfurt a.M. 1993, S. 13-33.

Hull, Margaret Betz: The Hidden Philosophy of Hannah Arendt, London/New York 2002.

Kovács, Gábor: »H. Arendt's Interpretation of Natural and Artificial in the Political Phenomenology of The Human Condition«, in: Limes: Borderland Studies $5\left({ }^{2} 2012\right)$, S. 93-102.

Olay, Csaba: »Cura rei publicae. Öffentlicher Raum und Macht bei Hannah Arendt«, in: M. Wischke (Hg.), Freiheit ohne Recht? Zur Metamorphose von Politik und Recht. Frankfurt a.M. 2011, S. 214-229.

Ricœur, Paul: Ricœur: Temps et récit 3. Le temps raconté, Paris 1985.

Rössler, Beate: Der Wert des Privaten, Frankfurt a.M. 2001.

Schindler, Roland: Geglückte Zeit - gestundete Zeit: Hannah Arendts Kritik der Moderne, Frankfurt a.M./New York 1996.

Schönherr-Mann, Hans-Martin: Hannah Arendt. Wahrheit, Macht, Moral, München 2006.

Trottmann, Christian: Faire, agir, contempler. Contrepoint à la Condition de l'homme moderne de Hannah Arendt, Paris 2008.

Szécsényi, Endre: »Remarks on Hannah Arendt’s Political Phenomenology«, in: L. Hagedorn/M. Staudigl (Hg.), Über Zivilisation und Differenz. Beiträge zu einer politischen Phänomenologie Europas, Würzburg 2007, S. 263-275.

Zaretsky, Eli: »Hannah Arendt and the Meaning of the Public/Private Distinction«, in: C. Calhoun/J. McGowan (Hg.), Hannah Arendt and the Meaning of Politics, Minneapolis/London 1997, S. 207-231.

Villa, Dana: Public Freedom, Princeton/Oxford 2008. 